\title{
Correction to: Novel smac mimetic APG- 1387 elicits ovarian cancer cell killing through TNF-alpha, Ripoptosome and autophagy mediated cell death pathway
}

Bao-Xia Li ${ }^{1 \dagger}$, Heng-Bang Wang ${ }^{2,3+}$, Miao-Zhen Qiu${ }^{4+}$, Qiu-Yun Luo ${ }^{1}$, Han-Jie Yi ${ }^{1}$, Xiang-Lei Yan ${ }^{1}$, Wen-Tao Pan ${ }^{1}$, Lu-Ping Yuan ${ }^{1}$, Yu-Xin Zhang ${ }^{1}$, Jian-Hua X U $^{2^{*}}$, Lin Zhang ${ }^{5^{*}}$ and Da-Jun Yang ${ }^{1,3^{*}}$

\section{Correction}

In the publication of this article [1], there was an error in the Methods, Cell cultures and reagents section: the Methods, Cell cultures and reagents section: 'The following primary antibodies were used: P62(\#8025), phospho-H2AX $(\gamma-\mathrm{H} 2 \mathrm{AX}$; \#9718), caspase-8(\#9746), RIP1(\#3493s), Beclin1(3738s), ATG7 (\#2631S), PARP (\#9546S), caspase-3(\#9665s), cIAP1 (\#7065s), cIAP2(3130s), XIAP(\#14334), FADD(\#2782S), phospho-NF-kBp105/p50(4806S), NF-kB2p100/p52(\#4882S) and TNFR1(\#3736S) were purchased from Cell Signaling Technology Inc.; GAPDH (\#sc-47724) from Santa Cruz Biotechnology (SC); LC3 (\#NB100-2220) from Novus Biologicals'.

Should instead read: 'The following primary antibodies: P62 (\#8025), phospho-H2AX ( $\gamma$-H2AX;\#9718), caspase-8 (\#9746), RIP1 (\#3493s), Beclin1 (3738s), ATG7 (\#2631S), PARP (\#9546S), caspase-3 (\#9665s), cIAP1 (\#7065), cIAP2 (3130s), XIAP (\#14334), FADD (\#2782S), NF-кBp105/p50 (4806S), NF-кB2p100/p52
(\#4882S), TNF- $\alpha$ (\#6945s), TNF- $\alpha$ neutralizing antibody (7321s), and TNFR1 (\#3736S) were purchased from Cell Signaling Technology Inc.; GAPDH (\#sc47724) was from Santa Cruz Biotechnology (SC); LC3 (\#NB100-2220) was from Novus Biologicals. Z-VADFMK (\#V116) and Necrostatin-1(\#N9037) were from Sigma. IKK-16(\#S2882) from Selleck'.

In the Results, APG-1387 is RIP1-dependent in ovarian cancer induced apoptosis section there was an error: 'We examined the protein levels of caspase-8/RIP1 by western blot, as shown in Fig. 4a'.

Should instead read: 'We examined the protein levels of caspase-8/RIP1 by western blot. APG-1387 triggered the activation of caspase- 8 and downregulated the protein level of RIP1, as shown in Fig. 4a'.

In the Results, APG-1387 induces apoptotic cell death through engagement of TNFR1 by TNF-alpha signaling pathway section there was an error: 'We have investigated the expression of NF-kB1/p50 and NF-kB2/p52 by

\footnotetext{
* Correspondence: xjh@fjmu.edu.cn; zhanglin@sysucc.org.cn; yangdj@sysucc.org.cn

${ }^{+}$Bao-Xia Li, Heng-Bang Wang and Miao-Zhen Qiu contributed equally to this work.

2Department of Pharmacology, Fujian Provincial Key Laboratory of Natural Medicine Pharmacology, School of Pharmacy, Fujian Medical University, Fuzhou 350108, China

${ }^{5}$ Departments of Clinical Laboratory, State Key Laboratory of Oncology in South China, Collaborative Innovation Center for Cancer Medicine, Sun Yat-Sen University Cancer Center, 651 Dongfeng Road East, Guangzhou 510060, China

${ }^{1}$ State Key Laboratory of Oncology in South China, Collaborative Innovation Center for Cancer Medicine, Sun Yat-sen University Cancer Center, Guangzhou 510060, China

Full list of author information is available at the end of the article
} 
western blot after cells were incubated with various concentrations of APG-1387'.

Should instead read: 'These results demonstrate that TNF $\alpha$ signaling is required for APG-1387-induced apoptotic cell death. Next, we have investigated the expression of NF- $\mathrm{kB} 1 / \mathrm{p} 50$ and NF- $\mathrm{kB} 2 / \mathrm{p} 52$ by western blot after cells were incubated with various concentrations of APG-1387'.

A section header contained an error: 'APG-1387-induced autophagy'

Should instead read: 'APG-1387 induces autophagy in ovarian cancer'

A section header contained an error: 'APG-1387-induced apoptosisby modulating autophagy'

Should instead read: 'Inhibition of autophagy sensitizes ovarian cancer cells to A PG-1387-induced apoptosis'.

In the Discussion section there was an error: 'APG1387 is a novel Smac mimetic. In our study, we investigated the molecular mechanisms underlying the inhibitory effect on the growth of ovarian cancer cell lines treated with varying concentrations of APG-1387'.

Should instead read: 'Restoring the apoptotic cell death machinery by pharmacological inhibition of IAPs proteins represents a compelling strategy for cancer therapy. APG-1387 is a novel Smac mimetic developed by Ascentage and currently being evaluated in phase I clinical trial. In our study, we investigated the in vitro and in vivo antitumor activity of APG-1387 in ovarian cancer'.

In the Discussion section there was an error: 'Our results suggest that APG-1387 induces autophagy during apoptosis. Autophagy plays a role in protecting cell survival. We have also found that it was effective as a single agent in vivo models. Treatment with APG-1387 induced potent cytotoxic and antiproliferative activity against established and human ovarian cancer cells'.

Should instead read: 'Our results suggest that APG1387 induces autophagy while triggering apoptosis. APG-1387-induced autophagy plays a role in protecting cell survival and inhibition of autophagy potentiates cytotoxicity of APG-1387 in ovarian cancer cells'.

Figure 7 caption had an an error: 'APG-1387-induced autophagy promote cell death in SKOV3 cells'.

Should instead read: 'Fig. 7 APG-1387-induced autophagy promotes cell survival in SKOV3 cells'.

In the Funding section there was an error due to the fact some additional funds were missing.

Should instead additionally read: 'the Project of International Science and Technology Cooperation of Fujian Province (Grant number. 2015I0002); and the Joint Funds for the innovation of science and Technology, Fujian province (Grant number. 2016Y91030065). The correct Figs. 2, 3 and 6 are presented here:

This has now been updated in the original article [1]. 


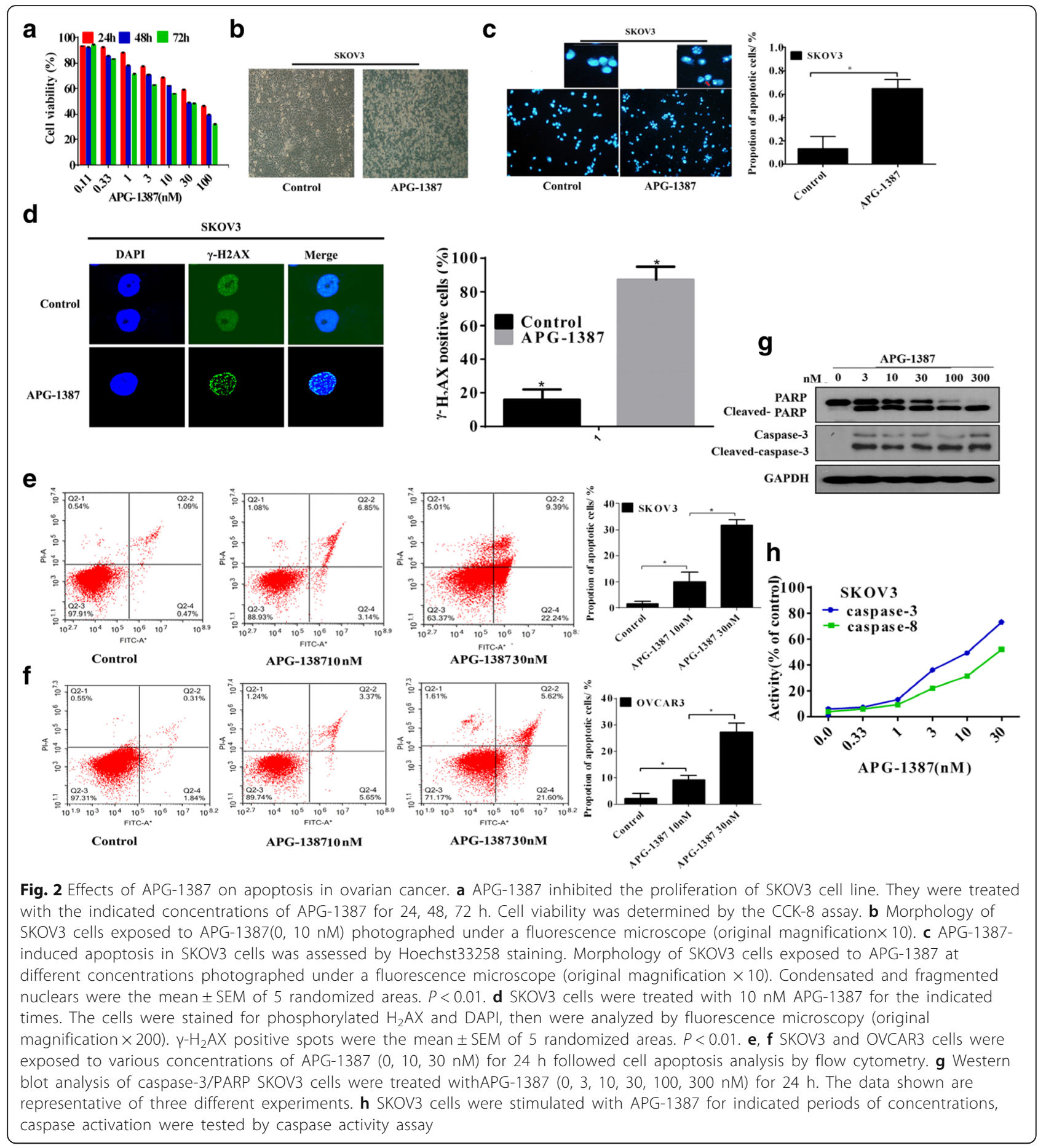


a

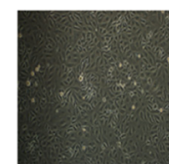

Control

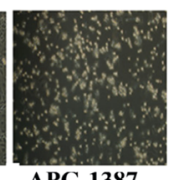

APG-1387

c

Control APG-1387 Z-VAD Z-VAD+APG-1387

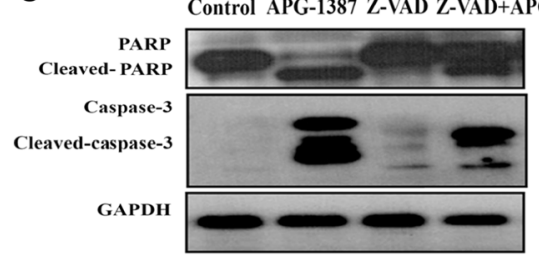

e

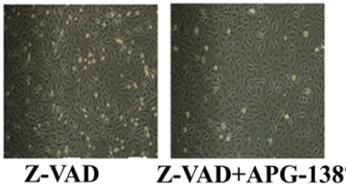

b
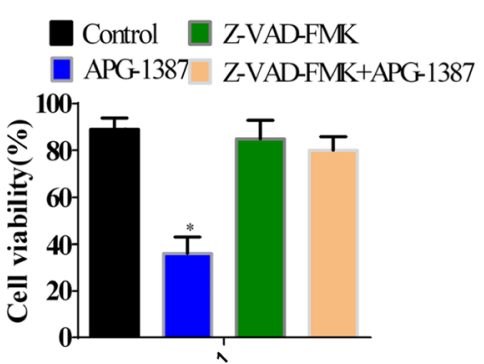

d

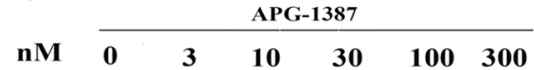

cIAP1

APG-1387

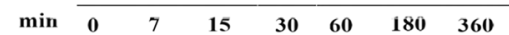

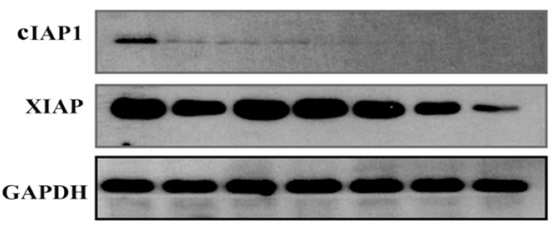

cIAP2

XIAP
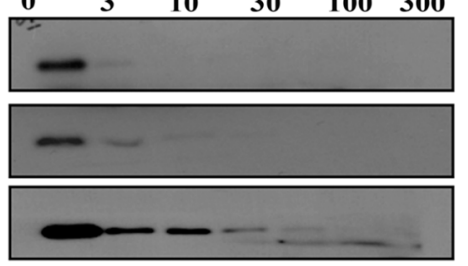

GAPDH

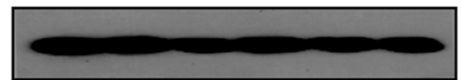

Fig. 3 APG-1387-induced apoptosis in caspase dependent manner. a Cells with or without addition of Z-VAD-FMK. Morphology of cells exposed to different treatment groups photographed under a fluorescence microscope (original magnification $\times 10$ ). b APG-1387 was coadministered with or without addition of caspase inhibitor (Z-VAD-FMK). Cell viability was determined by the CCK-8 assay. c Western blot analysis of the effect of APG-1387 with or without addition of Z-VAD-FMK on caspase-3/PARP expression level in SKOV3 cells. $\mathbf{d}$ Western blot analysis of the expression levels of IAPs at different concentrations of APG-1387 in SKOV3 cells. e Cells were treated with different time points, and the effect of APG-1387 on IAP family members expression level was determined by western blot. Data represent one of three experiments yielding similar results 

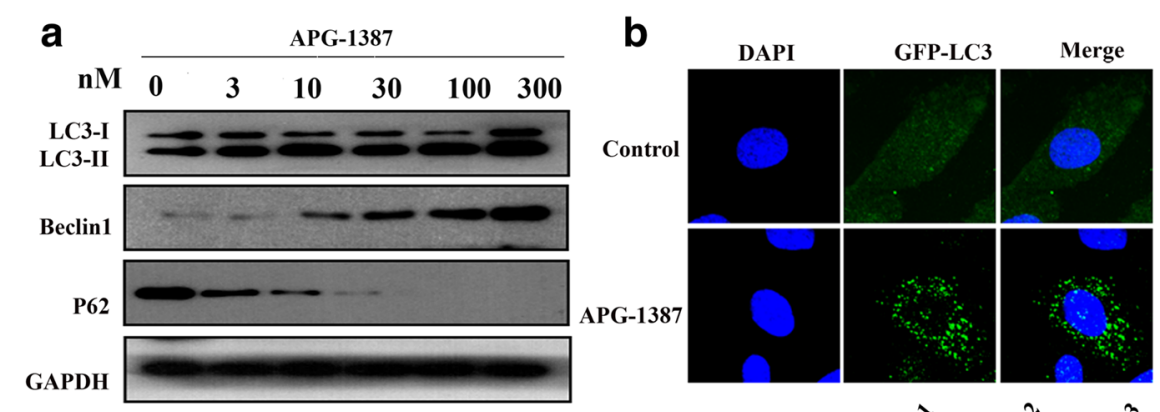

C

d
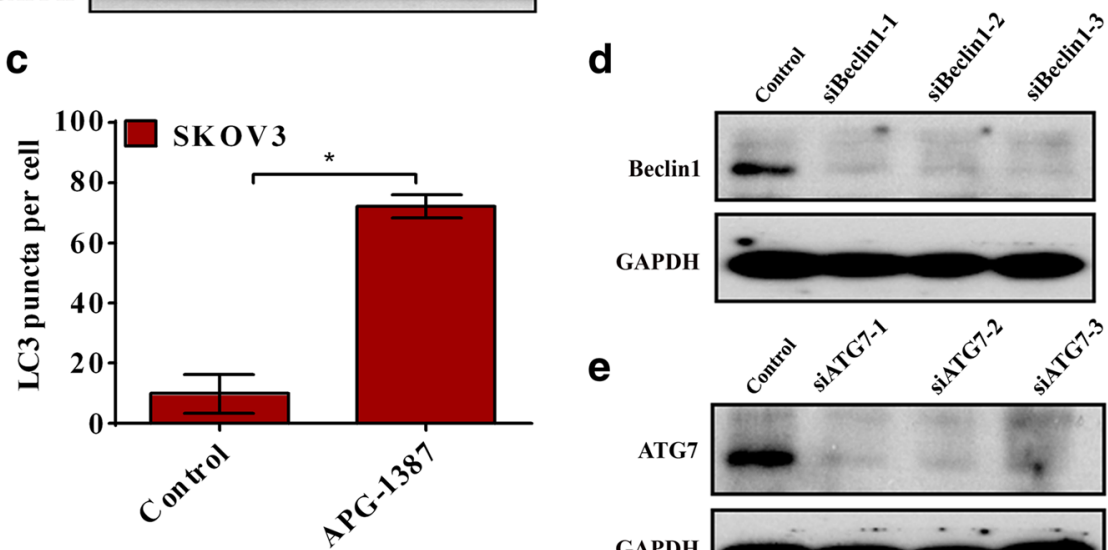

f

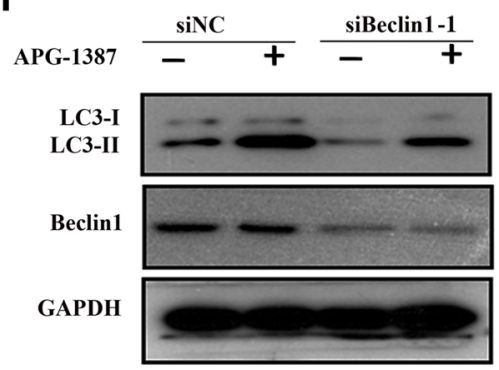

h

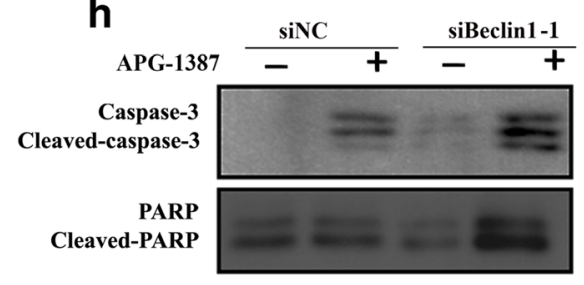

GAPDH

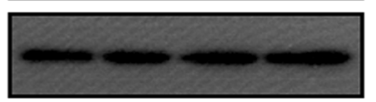

e
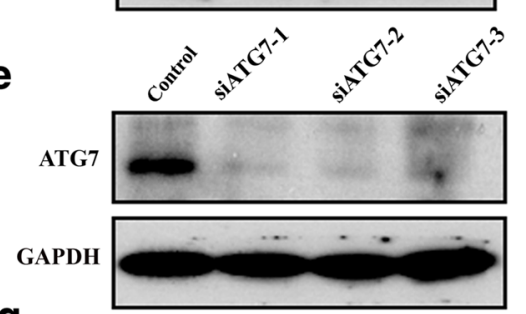

g APG-1387

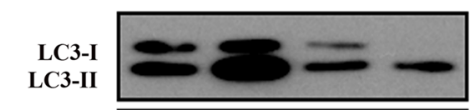

ATG7

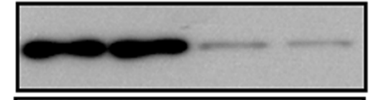

GAPDH

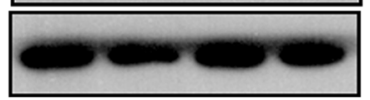

i

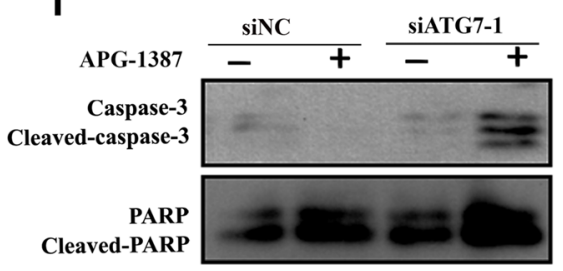

Fig. 6 APG-1387 induces autophagy in ovarian cancer cells. a The expression of LC3, Beclin1 and P62 was measured by western blot. Cells were treated with APG-1387(0, 3, 10, 30, 100, 300 nM) for 24 h. b Cells were transfected with GFP-LC3 plasmids, and then maintained in media with or without 3 nM APG-1387 for 24 h. The cells were then stained with DAPI and analyzed by fluorescence microscopy. c Statistical analysis of the percentage of LC3 puncta per cell. Columns, mean ( $n=3)$; bars, SD. * $P<0.01$ vs. untreated group. LC3 puncta per cell were quantified. $\mathbf{d}$ Cells were transfected with Beclin1 siRNAs. Western blot was used to detect the expression of Beclin1. e Cells were transfected with ATG7 siRNAs. Western blot was used to detect the expression of ATG7. f Cells were transfected with Beclin1 siRNAs. After $24 \mathrm{~h}$ treatment with or without 3 nM APG-1387, western blot analysis was performed for indicated proteins. $\mathbf{g}$ Cells were transfected with ATG7 siRNAs. After 24 h treatment with or without 3 nM APG-1387, western blot analysis was performed for indicated proteins. $\mathbf{h}$ Western blot analysis was performed for indicated proteins in cells transfected with siBeclin-1 and treated with 10 nM APG-1387. i Western blot analysis was performed for indicated proteins in cells transfected with siATG7-1 and treated with 10 nM APG-1387 


\section{Author details}

'State Key Laboratory of Oncology in South China, Collaborative Innovation Center for Cancer Medicine, Sun Yat-sen University Cancer Center,

Guangzhou 510060, China. ${ }^{2}$ Department of Pharmacology, Fujian Provincial Key Laboratory of Natural Medicine Pharmacology, School of Pharmacy, Fujian Medical University, Fuzhou 350108, China. ${ }^{3}$ Ascentage Pharma Group Corp., Ltd, Taizhou 225309, China. ${ }^{4}$ Department of Medical Oncology, State Key Laboratory of Oncology in South China, Collaborative Innovation Center for Cancer Medicine, Sun Yat-Sen University Cancer Center, Guangzhou

510060, China. ${ }^{5}$ Departments of Clinical Laboratory, State Key Laboratory of Oncology in South China, Collaborative Innovation Center for Cancer Medicine, Sun Yat-Sen University Cancer Center, 651 Dongfeng Road East, Guangzhou 510060, China.

Received: 14 March 2018 Accepted: 14 March 2018

Published online: 24 May 2018

\section{Reference}

1. Li BX, Wang HB, Qiu MZ, et al. Novel smac mimetic APG-1387 elicits ovarian cancer cell killing through TNF-alpha, Ripoptosome and autophagy mediated cell death pathway. J Exp Clin Cancer Res. 2018;37:53. https://doi. org/10.1186/s13046-018-0703-9 